\title{
The construction and application of self-cleaning engineering bacteria to control MBR biofilm fouling in suit
}

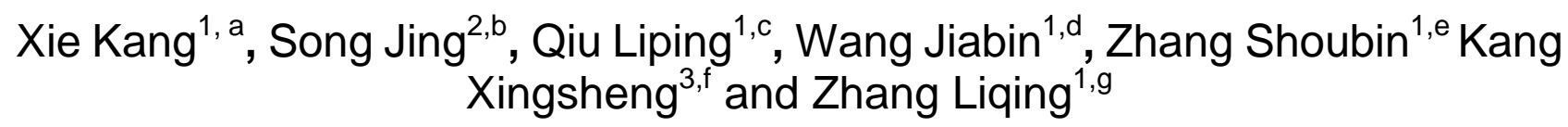

${ }^{1}$ School of Civil Engineering and Architecture, University of Jinan, Jinan, Shandong 250022, China

${ }^{2}$ Shandong Province Environmental Protection Technology Service Center, Jinan, Shandong 250102, China

\author{
${ }^{3}$ Shandong Academy of Environmental Science, Jinan, Shandong 250013, China \\ acea_xiek@ujn.edu.cn, ${ }^{b}$ amay8369@163.com, ccea_qiulp@ujn.edu.cn, ${ }^{\mathrm{d}}$ cea_wangjb@ujn.edu.cn, \\ ece_zhangsb@ujn.edu.cn, ${ }^{\mathrm{i}}$ xingshengkang@163.com, ${ }^{9} \mathrm{cea}$ chhanglq@ujn.edu.cn
}

Keywords: double membrane bioreactor, forward osmosis, ultrafiltration, removal of nitrogen and phosphorus.

Abstract. The biofilm fouling is the main factor for MBR fouling. Conventional control methods are difficult to mitigate membrane fouling for a long time. Other methods like quorum sensing, biofilm disperse protein, NO, etc. are not control MBR biofilm fouling in suit. So, combining the functional gene to constructe a self-cleaning engineering bacteria can alleviate the the formation of biofilm on the membrane surface effectively. It provides a new idea and supportion to control MBR biofilm fouling.

\section{Introduction}

Pollution and scarcity of water resources have become an important factor which restricts the development of human society. The traditional sewage biological treatment technology cannot meet the increasingly stringent emission and reuse standards. Membrane bioreactor (MBR) technology combines membrane filtration and biological treatment technology, which effectively improves the effluent water quality and treatment efficiency. It has attracted more and more attention in recent years and has been widely used in municipal wastewater treatment, industrial wastewater treatment and reuse. However, the biological fouling (is mainly for biofilm fouling which produced by microorganisms and metabolites on the membrane surface deposition, growth) in MBR always lead to decline membrane flux and increasing energy consumption and maintenance cost. It has seriously affected the economy and practicability of MBR technology, and restricted its large-scale development. Therefore, how to effectively alleviate the formation of biofilm on membrane surface is the key problem to guarantee the long-term stable operation of MBR.

The traditional methods of membrane fouling control mainly focus on membrane cleaning, operation process optimization and membrane material modification. Membrane cleaning is mainly divided into physical, chemical and physical chemistry, combined cleaning, etc. Physical / chemical cleaning methods (including backlash, ultrasonic, mechanical cleaning, shock and chemical cleaning) can only temporarily reduce membrane fouling. It is easy to damage the inherent characteristics of the membrane, and the released chemicals compounds by chemical cleaning and the cleaning by-products are likely to infect the system microorganisms. These not only destroy the biological stability, but also pose a potential threat to the water environment safety [1].

The optimization of the operation process is mainly to control the formation of MBR biofilm pollution by changing the operating conditions of MBR and then affecting the characteristics of mixing liquid in MBR. The more common way is to dose biological carriers or poly substance to the system[2], or to form granular sludge, etc. In addition, control the formation of biofouling with the addition of electric field or magnetic field [3] has been attracted more attention in recent years. 
It is the hot topic of membrane material modification to control biofouling. It is mainly through matrix modification or surface modification methods such as the introduction of polar groups into the membrane material to increase membrane hydrophilicity, so as to reduce the formation of biofouling.

The above biofilm fouling control technology indirectly control the growth of the biofilm on the membrane surface by changing the external conditions. So, it is difficult to fundamentally solve the biofilm formation on membrane surface. In recent years, the formation and control of bacterial biofilm have been studied deeply in bacterial resistance, which provides a reference for MBR biofilm control.

In this study, we try to find a in-suit technology to control the membrane biofilm fouling in MBR.

\section{Experimental design}

The recent research shows that the formation and dispersion process of biofilm are regulated by environmental factors. This regulation mainly affected through the quorum sensing (QS) and / or the second messenger cyclic guanosine diphosphate (c-di-GMP). QS has received extensive attention and deep research in the formation and control of biofilm [4]. Quorum sensing among bacteria plays an important role in the formation and control of biofilms during water and wastewater treatment[5]. It has also been applied to analyze the interaction between quorum sensing and biofilm formation in MBR systems, in order to explore an in-situ control of MBR biofilm fouling. However, the effect of formation and quenching mechanism of quorum sensing on MBR biofilm fouling is still unclear and need to do further study.

In recent years, the control of MBR biofilm fouling by quorum quenching has been studied. The encapsulated immobilized enzyme is used to dose into the MBR by inducing quorum quenching to control the biofilm formation on the membrane surface. At present, the technology of quorum quenching to control biofilm fouling still requires complex immobilization pretreatment. The extraction/screening cost of quenching materials is still higher, and the development of quenching technology still need to be further optimized. At the same time, the immobilized quenching material directly into MBR will easily lead to disperse by the gas water stir. This is not conducive to the capture of signal molecules. Thus, it will reduce its ability to inhibit membrane surface biofilm formation. Therefore, it is also an important problem for the efficient application of quorum quenching to control the biofilm formation nearto membrane surface.

It has attracted widespread attention in biofilm dispersing inducer without quorum quenching to control biofilm formation on the membrane surface. It is shown that dispersed proteins, cis-2-decanoic (acid) and nitric oxide (NO) can interfere with intracellular signaling substances, and then effectively disperse biofilms. With the development of gene engineering technology, inserting harmless gene fragments into bacteria and transforming the expression of biofilm dispersed proteins can effectively induce the dispersion of biofilm. Ma et. al. [6] found that the engineered protein BdcA can effectively bind c-di-GMP, reducing its concentration level, which can disperse the biofilm on membrane surface.

NO is a common signaling molecule in biological systems. It can promote the phosphodiesterase (PDE) activity to improve the degration of c-di-GMP, which also can reduce the concentration of c-di-GMP. So, the NO can induce the biofilm dipersion.

Combining the functional gene of quorum sensing, biofilm dispersion protein and NO functional gene structure the engineering bacteria. This engineering bacteria can regulate growth by itself responding to the cell density on membrane surface and disperse other biofilm. This provides a new methods for the self regulation of MBR fouling and makes it possible to control MBR biofilm fouling in situ.

The Pseudomonas aeruginosa cell LasI/LasR QS module is combined with the biofilm dispersed proteins Hha or BdcA, inserting the functional gene nirS or NOS synthetase. And then trasformate these gene fragments into Escherichia coli. A self cleaning engineering bacteria could be constrcted. The self-cleaning engineering bacteria is shown in Fig. 1. 


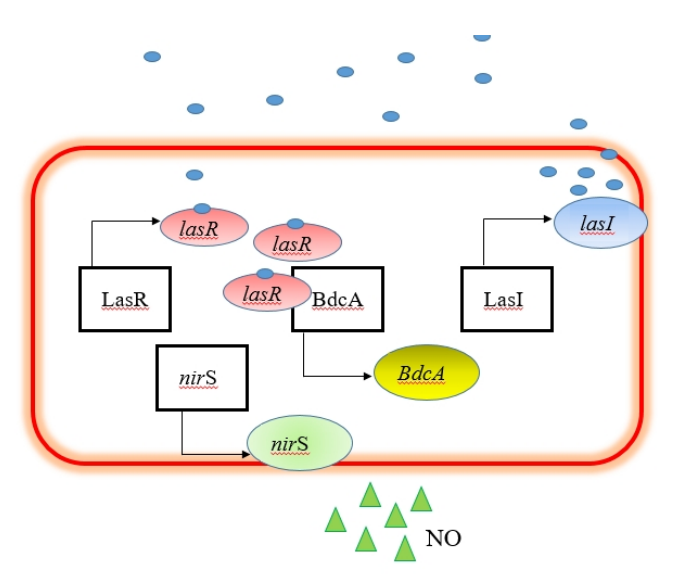

Fig. 1 The construction of self-cleaning engineering bacteria

However, the effective growth of self-cleaning engineering bacteria on the MBR membrane surface is the key issue to control biofilm fouling. Traditional direct dosing methods are susceptible to gas water washing and the effects of indigenous microorganisms on the system. Although the immobilization technology can overcome the above problems, it will cause engineering bacteria to spread out in the system, which is not conducive to the production of NO to disperse the biofilm. Therefore, it is important to find an efficient application technology of self-cleaning engineering bacteria to control the MBR biofilm fouling. The MBR membrane module is effectively reformed through integrated tube bundle and process optimization. This can form an aqueous flow film on the membrane surface. It not only enhances the growth of self-cleaning engineering bacteria, but also effectively resists the effects of air, water erosion and indigenous microorganisms threaten. The integrated tube bundle is shown in Fig.2.

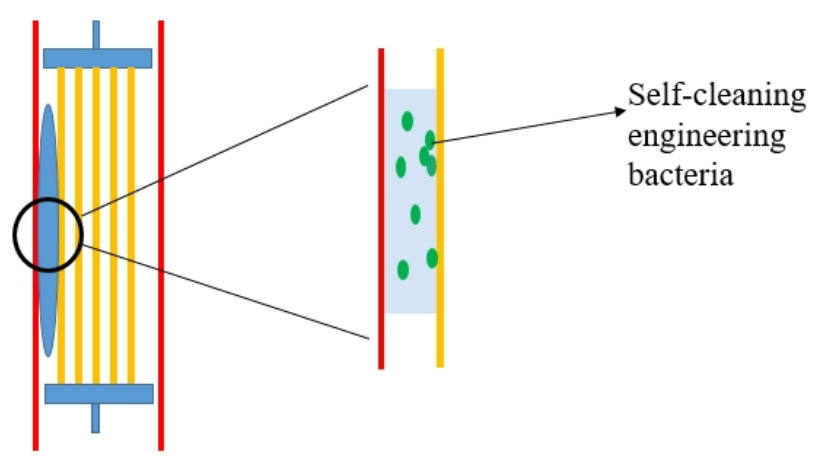

Fig. 2 The integrated tube bundle

In order to do further study of self-cleaning engineering bacteria to control MBR biofilm fouling, the following three aspects have become the focus. It should be investigated clearly the effect mechanism and influencing factors of MBR microbial quorum sensing on membrane biofilm firstly. And then, the construction and expression of self-cleaning engineering bacteria and their physiological and biochemical characteristics should be studied. Lastly, optimization analysis of the application of self-cleaning engineering bacteria to control MBR membrane fouling in situ should also be explored.

\section{Conclusions}

The relationship between the change of microbial population, the formation trend of biofilm fouling and the quorum sensing affection was studied in MBR. It should be elucidated the mechanism and key influencing factors of biofilm formation which caused by quorum sensing. And then, the self-cleaning engineering bacteria can be constructed. Through the optimization of technological 
conditions, the technical methods for the effective application of self-cleaning engineering bacteria in MBR were established lastly.

\section{Acknowledgements}

This work was financially supported by the research foundation of University of Jinan (XKY1618), Science and technology plan project of Shandong higher education institutions (J17KA198), National Natural Science Foundation of China (51678726, 51278225, 51408260), Science and Technology Development Projects of Shandong Province (2016GGB01157), Shandong Provincial Natural Science Foundation (ZR2013EEQ007, ZR2015EM021).

\section{References}

[1] L.J. Deng, W.S. Guo, H.H. Ngo, H.W. Zhang, J. Wang, J.X. Li, S.Q. Xia, Y. Wu: Bioresource Technology, Vol. 221 (2016), p.656.

[2] J. Ji, J.F. Li, J.P. Qiu, X.X. Li: Separation and Purification Technology, Vol. 131 (2014), P.1

[3] J. Zhang, A. Satti, X.G. Chen, K. X, J.Y. Sun, X.X. Yan, P. Liang, X.Y. Zhang, X. Huang: Chemical Engineering Journal, Vol. 273 (2015), P.223

[4] Ky.M. Yeon, W.S. Cheong, H.S. Oh, W.N. Lee, B.K. Hwang, C.H. Lee, H. Beyenal, Z. Lewandowski: Environmental Science Technology, Vol. 43(2009), P.380

[5] J.H. Huang, Y.H. Shi, G.M. Zeng, Y.L. Gu, G.Q. Chen, L.X. Shi, Y. Hu, B. Tang, J.X. Zhou: Chemosphere, Vol. 157(2016), P.137

[6] Q. Ma, G.S. Zhang, T.K. Wood: BMC Research Notes, Vol. 4(2011), P.447 